Artículo

\title{
Tolerancia a estrés hídrico en frijol cv. Pinto Saltillo modificado con el gen pirofosfatasa vacuolar-1 de Arabidopsis thaliana
}

\author{
David Cadena-Hernández ${ }^{1}$ \\ Roberto Gaxiola-Ariza ${ }^{2}$ \\ María Alejandra Mora-Avilés ${ }^{3 \S}$ \\ ${ }^{1}$ Ingeniería Bioquímica-Instituto Tecnológico de Celaya. Celaya, Guanajuato, México. (davidcadena. \\ ibq@ hotmail.es). ${ }^{2}$ School of Life Sciences-Arizona State University. Tempe, USA. AZ. 85287. ${ }^{3}$ Ingeniería \\ en Biotecnología-Universidad Politécnica de Guanajuato. Av. Universidad Sur 1001, Localidad Juan \\ Alonso, Cortazar, Guanajuato, México. CP. 38483. \\ ${ }^{\S}$ Autora para correspondencia: mora_alejandra@yahoo.com.
}

\section{Resumen}

El gen Arabidopsis Vacuolar Pyrophosphatase-1 (avp1) por sus siglas en inglés, el cual codifica a la enzima $\mathrm{H}+$ pirofosfatasa mejora la tolerancia a sequía y salinidad e incrementa la biomasa y rendimiento cuando se sobreexpresa en plantas transgénicas. El propósito de este estudio fue analizar las características fenológicas, fisiológicas y agronómicas del frijol cv. Pinto Saltillo (PS) transformado con el gen 35Sprom:avp1 bajo condiciones de estrés hídrico extremo. El diseño experimental consistió en una distribución aleatoria de 10 plantas homocigotas por cada una de las 9 líneas PS-avp1, así como plantas control no modificadas. El riego fue suspendido en la etapa fenológica de $50 \%$ de floración y el estrés hídrico inició a partir del $100 \%$ hasta $9 \%$ de contenido volumétrico de agua (CVA), a lo largo de 10 días. Los resultados mostraron que el índice de intensidad de sequía (IIS) fue de 0.78. Aun cuando la conductancia estomática y la transpiración mostraron una tasa de disminución similar a las plantas no transformadas, la tasa fotosintética de todas las líneas PS-avp1 fue superior a las plantas control bajo condiciones de estrés hídrico. El aumento de la biomasa total en algunas líneas PS-avp1 no correlacionó con un rendimiento mayor. Siete de las nueve líneas evaluadas mostraron un mayor rendimiento (35\% - 96\%) comparadas con las plantas PS no transformadas bajo condiciones de estrés, indicando una mayor eficiencia en la translocación de fotoasimilados a los órganos destino (semillas).

Palabras clave: componentes de partición, rendimiento, tolerancia a sequía.

Recibido: abril de 2019

Aceptado: julio de 2019 


\section{Introducción}

El frijol (Phaseolus vulgaris L.) es un cultivo básico importante el cual es consumido por aproximadamente 400 millones de personas en los trópicos (CIAT, 2017). El origen y diversidad de $P$. vulgaris antes de la domesticación, se remonta a dos regiones principales: la Mesoamericana y la Andina, donde el frijol adquirió varias características agronómicas (Gepts, 1998). Las razas en el grupo Mesoamericano (Mesoamericana y Durango) muestran secuencias genéticas asociadas a la tolerancia a estrés hídrico (Terán y Singh, 2002). Hay dos tipos diferentes de estrés hídrico, el intermitente y el terminal.

El estrés intermitente es causado por patrones climáticos de lluvia esporádica lo cual resulta en intervalos de sequía que pueden ocurrir en cualquier momento durante el proceso de desarrollo (Schneider et al., 1997). En contraste, la sequía terminal tiene lugar cuando la planta atraviesa por estrés hídrico durante los estadios reproductivos y es la decisiva y contundente en la producción y rendimiento. La eficiencia de algunos métodos de mejoramiento para tolerancia a estrés hídrico, tales como la selección asistida por marcadores moleculares, ha tenido resultados limitados.

Esto ha sido debido a los genotipos con bajo nivel de polimorfismo, presencia de recombinación gen-marcador, resolución variable de mapeo de QTL y ocurrencia de interacciones genotipoambiente (Villordo-Pineda et al., 2015). Todos estos son factores importantes requeridos para la identificación de variabilidad genética en la población, con el objetivo de identificar los mejores marcadores genéticos. La evidencia indica que el uso de ADN recombinante tiene oportunidad en el mejoramiento de cultivos como el frijol (Espinosa et al., 2013), permitiendo generar plantas tolerantes a estrés hídrico.

El gen recombinante avp1 de Arabidopsis thaliana, asociado a la tolerancia y estrés hídrico, codifica para el polipéptido tipo I de la H+-pirofosfatasa de cerca de $80 \mathrm{KDa}$ (Maeshima y Yoshida, 1989; Sarafian et al., 1992). La sobreexpresión del gen avp1 ha resultado en tolerancia al estrés hídrico y salino en A. thaliana (Gaxiola et al., 2001), tomate (Park et al., 2005), alfalfa (Bao et al., 2008), maíz (Li et al., 2008), algodón (Pasapula et al., 2011), maní (Qin et al., 2013), lechuga y arroz todos ellos con fenotipo similar como mayor biomasa de brotes, raíces y mayor rendimiento.

El objetivo de este estudio fue evaluar las caracterísiticas agronómicas, fisiológicas y fenológicas de líneas de frijol cv. Pinto Saltillo transformado con el gen 35Sprom:avp1 bajo condiciones severas de sequía terminal en condiciones de invernadero.

\section{Materiales y métodos}

El casete de expresión 35Sprom-avp1 fue previamente diseñado por Gaxiola et al. (2001). El plásmido fue introducido en Agrobacterium tumefaciens cepa GV2260 vía choque térmico (Höfgen y Willmitzer, 1988) y posteriormente usada para transformar frijol cv. Pinto Saltillo (SánchezValdez et al., 2004).

Transformación de frijol. La esterilización superficial, germinación de semilla, disección de hipocotilos y componentes del medio fueron descritos por Espinosa-Huerta et al. (2013). El medio líquido de inducción (MI) consistió de medio B5 (Gamborg et al., 1968) [suplementado con mio- 


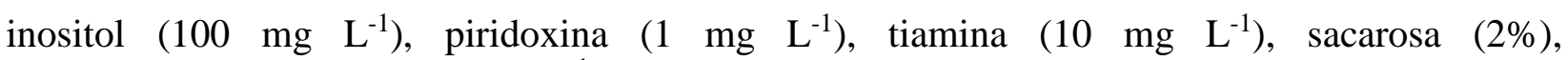
bencilaminopurina-HC1 (10 $\mathrm{mg} \mathrm{L}^{-1}$ ) (Sigma-Aldrich), a $\left.\mathrm{pH} 5.8\right]$, fue añadido al cultivo de $A$. tumefaciens, cepa GV2260 (O.D.600=0.8) en una proporción 5:1 (v/v).

Los hipocotilos fueron incubados por 10 min en esta solución con movimientos circulares suaves. Los explantes fueron cultivados en medio de co-cultivo sólido, el cual consistió de MI adicionado con $200 \mathrm{mM}$ de acetociringona (3', 5' -Dimetoxi-4'-hidroxiacetofenona) (Sigma-Aldrich) y Agar (6 $\mathrm{mg} \mathrm{L}^{-1}$ ) (tipo A, para cultivo de células vegetales) (Sigma-Aldrich) y fueron incubados por $5 \mathrm{~d}$ a $25^{\circ} \mathrm{C}, 16 \mathrm{~h}$ luz $\left(45-70 \mathrm{mmol} \mathrm{m}^{-2} \mathrm{~s}^{-1}\right)$ y $8 \mathrm{~h}$ oscuridad.

Posterior al co-cultivo, los hipocotilos fueron transferidos a medio de eliminación de Agrobacterium el cual consistió de MI sólido adicionado con $300 \mathrm{mg} \mathrm{L}^{-1}$ de timentina $\left(\right.$ GlaxoSmithKline $^{\circledR}$ ) y permanecieron en este medio por $5 \mathrm{~d}$ bajo las mismas condiciones del cultivo. Posteriormente, los explantes fueron transferidos a medio de selección con los mismos componentes del medio de eliminación, adicionado con $50 \mathrm{mg} \mathrm{L}^{-1}$ de kanamicina (Sigma-Aldrich).

Los brotes diferenciados que permanecieron verdes en medio de selección fueron separados del explante original (hipocotilo) y cultivados individualmente en cajas Magenta ${ }^{\circledR}$ conteniendo medio de elongación y enraizamiento (MI sin reguladores de crecimiento), adicionado con $50 \mathrm{mg} \mathrm{L}^{-1} \mathrm{de}$ kanamicina y $300 \mathrm{mg} \mathrm{L}^{-1}$ de timentina para promover el crecimiento de la plántula y la formación de raíz. Las condiciones de cultivo fueron idénticas a los estadios previos.

Las plántulas regeneradas in vitro se llevaron a macetas con sustrato Sunshine ${ }^{\circledR}$ Universal Mix (Sun Gro Horticulture Canada Ltd.) y aclimatadas a condiciones in vivo. Estas crecieron en invernadero a $25-28{ }^{\circ} \mathrm{C}$ y una intensidad de luz de 170 a $285 \mathrm{mmol} \mathrm{m}^{-2} \mathrm{~s}^{-1}$ hasta la producción de semilla.

\section{Material vegetal}

Nueve líneas homocigotas de frijol cv. Pinto Saltillo (PS) generación T4 modificadas con una copia del casete de expresión 35Sprom-avp1 de acuerdo al análisis de segregación (L1, L5, L7, L9, L10, L11, L12, L13 and L14) (PS-avp1), así como los comparadores no modificados del mismo fondo genético PS, fueron evaluados en bioensayos de estrés hídrico en condiciones de invernadero.

\section{Caracterización molecular de líneas de frijol PS-avp1}

El DNA de cada línea fue aislado de acuerdo con el protocolo de Murray and Thompson (1980) empleando 100-200 mg de tejido foliar por muestra para identificar vía PCR los elementos de la construcción insertada y confirmar su estado de planta transformada genéticamente.

Las reacciones de amplificación por PCR punto final consistieron de muestras de DNA (50 ng), iniciadores $(0.2 \mathrm{mM})$, dNTP $(0.25 \mathrm{mM})$, Taq polimerasa $(1 \mathrm{U})$, cloruro de magnesio $(2 \mathrm{mM}) \mathrm{y}$ solución amortiguadora Taq (1X) (10 mM Tris-HCl, $50 \mathrm{mM} \mathrm{KCl,} 1.5 \mathrm{mM} \mathrm{MgCl}$, pH 8.3). Los iniciadores de la secuencia del gen avp1 consistieron de avp1 sentido, 5'-GGC TCT GTT GAG GGA TTC AG-3' y avp1 antisentido, 5'-GCA ATG ACA GCT GGG TTT CTT-3', las condiciones de amplificación fueron 10 min a $95{ }^{\circ} \mathrm{C}$ durante un ciclo, seguido por 30 ciclos de desnaturalización a $95{ }^{\circ} \mathrm{C}$ por $1 \mathrm{~min}$, alineamiento a $55^{\circ} \mathrm{C}$ por $30 \mathrm{~s}$ y extensión a $72{ }^{\circ} \mathrm{C}$ por $1 \mathrm{~min}$, además de una extensión final a $72{ }^{\circ} \mathrm{C}$ por $10 \mathrm{~min}$ (Pasapula et al., 2011). Los iniciadores utilizados no amplificaron ningún fragmento endógeno de frijol no transformado. 


\section{Cuantificación de la expresión génica}

El tejido de hoja fue colectado de plantas en estadio fenológico de $50 \%$ de floración, y para la extracción de ARN total se empleó el agente comercial Trizol ${ }^{\circledR}$ (Reagent, Carisbad, CA, USA), siguiendo las instrucciones del fabricante. El ARN total fue cuantificado por espectrofotometría (Nanodrop). El análisis de expresión por PCR cuantitativo fue realizado con el termociclador tiempo real ABI PRISM 7000 SDS (Applied Biosystems). Los oligonucleótidos y la sonda fueron diseñados usando el software Primer Express versión 2.0 (Applied Biosystems) y basado en la secuencia del gen avp1 (NCBI, NM_001084073).

Estos consistieron en iniciador avp1 sentido 5' - AGT GCA GCT CTT AAG ATG GTT GAA G 3', iniciador avp1 antisentido 3' - ATG AGT CCA GGG ATG GTG TTG - 5, y sonda AAC TGC CTG CGA ACT T marcada con el fluorocromo FAM ${ }^{\mathrm{TM}}$. El control endógeno fue el gen ribosomal 18S (4319413E, Applied Biosystems) marcado con el fluorocromo VIC ${ }^{\text {TM }}$. La síntesis de ADNc fue por transcripción en reversa (RT-PCR) usando One-Step RT-PCR Master Mix de Applied Biosystems (Cat. No. 4309169). Cada reacción de $25 \mu \mathrm{L}$ consistió de $12.5 \mu \mathrm{L}$ de TaqMan ${ }^{\circledR} 2 \mathrm{x}$ Universal PCR Master Mix No AmpErase ${ }^{\circledR}$ UNG, $0.625 \mu \mathrm{L}$ de 40X MultiScribe y mezcla de inhibidor de ARNasa, 1.25 $\mu \mathrm{L}$ de la sonda del gen avp1, 1.25 $\mu \mathrm{L}$ de la sonda del gen ribosomal $18 \mathrm{~S}$ y $25 \mathrm{ng}$ of ARN total.

Las condiciones de amplificación consistieron en 30 min a $48^{\circ} \mathrm{C}$ (transcripción en reversa), 10 min a $95{ }^{\circ} \mathrm{C}$ (desnaturalización) y 45 ciclos (amplificación): $95{ }^{\circ} \mathrm{C}$ por $15 \mathrm{~s} \mathrm{y} 60{ }^{\circ} \mathrm{C}$ por 1 min. Los resultados de amplificación fueron analizados usando el método comparativo $\Delta \mathrm{Ct}$, el cual normaliza las lecturas de avp1 con las lecturas del control endógeno $18 \mathrm{~S}$ de tal manera que las diferencias entre niveles de expresión serán únicamente debido a la sobreexpresión del gen en el tejido y no a variaciones en el $\mathrm{ARN}$ experimental $(\Delta \mathrm{Ct}=\mathrm{Ct}$ avp1-Ct $18 \mathrm{~S})$. Posteriormente, cada $\Delta \mathrm{Ct}$ de las réplicas fue comparado con el valor más alto o calibrador $[\Delta \Delta \mathrm{Ct}=\Delta \mathrm{Ct}$ (muestra) $-\Delta \mathrm{Ct}$ (calibrador)]. Finalmente, el gen avp1 vs la expresión relativa fue generada elevando el valor de la muestra al cuadrado menos el calibrador (2- $\Delta \Delta \mathrm{Ct}$ ) (Livak and Schmittgen, 2001).

\section{Tratamiento de estrés hídrico}

Diez semillas de cada línea PS-avp1 y 20 semillas de frijol PS no modificado, fueron germinadas en macetas de $2.5 \mathrm{~L}$ con $30 \%$ de sustrato Sunshine ${ }^{\circledR}$ y $70 \%$ de vermiculita. El sustrato fue mantenido a capacidad de campo o $100 \%$ de contenido volumétrico de agua (CVA). Las plántulas fueron fertilizadas en dos ocasiones durante el proceso de crecimiento (floración e iniciación de llenado de semilla) con $3 \mathrm{~g}$ de urea. El tratamiento de estrés hídrico inició en el estado fenológico de $50 \%$ de floración, de $100 \%$ hasta $9 \%$ de CVA (10 días).

A partir, de 9\% de CVA las plantas fueron tratadas con riego de recuperación diario hasta que el llenado de vaina fue completado. El frijol PS no modificado en condiciones de riego o bajo estrés hídrico fue utilizado como control. El experimento fue establecido en el Campo Experimental Bajío del INIFAP durante la primavera de 2014, bajo condiciones confinadas. Las condiciones medioambientales en el invernadero consistieron en una temperatura mínima de $18{ }^{\circ} \mathrm{C}$ durante la noche y una máxima de $35^{\circ} \mathrm{C}$ durante el día. La luminosidad natural ocurrió; a través, de techo de cristal y la humedad relativa fue de $70 \%$. 


\section{Diseño experimental y análisis estadístico}

El diseño experimental de la evaluación fenológica, fisiológica y agronómica fue de bloques completos, cada línea PS-avp1 y el control PS representaron un bloque. La unidad experimental consistió en una maceta, la cual contenía una planta y se emplearon diez réplicas por línea o material control. Los datos reunidos fueron sujetos a un análisis de varianza y las diferencias entre medias fueron comparadas usando la prueba de Tukey $(p \leq 0.05)$. Los coeficientes de correlación (r) entre variables relevantes también fueron calculadas.

\section{Variables fisiológicas}

La evaluación de las variables fisiológicas fue llevada a cabo usando el sistema portátil de fotosíntesis CID-340 (CI-340 Handheld Photosynthesis System. Bio-Science), el cual fue empleado para registrar la tasa fotosintética, la conductancia estomática y la tasa de transpiración. Las lecturas de estas variables fueron tomadas en hojas jóvenes y suculentas del $2^{\circ}$ estrato de la planta, tanto en poblaciones bajo estrés como en riego. Las lecturas fueron tomadas diariamente por 10 da la misma hora del día, con el fin de reducir los errores derivados de variaciones en la incidencia solar.

\section{Componentes fenológicos}

La variable días a floración (DF) fue registrada cuando el $50 \%$ de las plantas de la población exhibieron al menos una flor abierta, mientras que los días a madurez fisiológica (DMF) fueron registrados cuando $75 \%$ a $90 \%$ de las vainas perdieron su pigmentación verde. Las variables fueron registradas diariamente hasta que el cambio del estadio fenológico fue identificado. La variable días a llenado de semilla (DLS) fue calculada usando la expresión DLS=DMF-DF.

\section{Rendimiento}

El rendimiento del cultivo (g) fue representado como el peso de todas las semillas cosechadas de las diez plantas evaluadas por línea y por la población PS control.

\section{Componentes de biomasa}

Cuando las plantas de cada línea PS-avp1 y población de frijol PS alcanzaron la madurez fisiológica fueron seccionadas en hojas, tallos, raíces, vainas y semillas de todas las plantas. Los tejidos, excepto las semillas fueron deshidratados completamente a $50{ }^{\circ} \mathrm{C}$ por $72 \mathrm{~h}$ y tanto el peso fresco como el peso seco de cada tejido fueron registrados. Los datos de la biomasa por tejido (g) y biomasa total ( $\mathrm{g}$ ) fueron colectados por tratamiento (estrés y no estrés) basado en los bloques (10 plantas).

\section{Componentes de partición}

Las variables de partición se refieren a los índices de selección que involucran rendimiento, biomasa, días a madurez fisiológica y días a llenado de semilla y determinan la eficiencia de las plantas en la translocación de fotosintatos a los órganos destino. Estos índices fueron calculados empleando las siguientes expresiones. 
Tasa de crecimiento de biomasa (TCB) se refiere al tiempo promedio de incremento total de biomasa en relación a los días en que la planta alcanzó su madurez fisiológica.

$\mathrm{TCB}=\frac{\text { Biomasa total }}{\mathrm{DMF}}$

La tasa de crecimiento económico (TCE) está definida como la eficiencia del rendimiento del cultivo en un tiempo determinado.

$\mathrm{TCE}=\frac{\text { Rendimiento }}{\mathrm{DMF}}$

La Tasa de Crecimiento de Semilla (TCS) indica el efecto de las condiciones existentes entre el estado de floración y el inicio de llenado de semilla.

$\mathrm{TCS}=\frac{\text { Rendimiento }}{\text { DLS }}$

El índice de cosecha (IC) se refiere a la eficiencia de la energía metabólica utilizada por el cultivo para sintetizar los productos orgánicos necesarios para su desarrollo.

$\mathrm{IC}=\frac{\text { Rendimiento }}{\text { Biomasa total }}$

La fuerza relativa de demanda (FRD) está relacionada con las emisiones de $\mathrm{CO}_{2}$ e indica la cantidad de energía necesaria para el desarrollo de órganos en la planta. Las líneas con alta tasa de FRD en cualquier entorno lumínico dado serían capaces de tener mejor respuesta a las emisiones de $\mathrm{CO}_{2}$.

$\mathrm{FRD}=\frac{\mathrm{TCS}}{\mathrm{TCB}}$

El rendimiento medio geométrico $(\mathrm{G})$ emplea el rendimiento de cada línea PS-avp1 en condiciones de estrés hídrico y riego para calcular el efecto y la intensidad del estrés hídrico en el rendimiento (Samper y Adams, 1985).

$\mathrm{G}=\left(\mathrm{Y}_{\mathrm{d}} \times \mathrm{Y}_{\mathrm{p}}\right)^{1 / 2}$

Donde: $Y_{d}$ y $Y_{p}$ se refieren al rendimiento o desempeño de cada línea bajo estrés y riego, respectivamente.

El índice de intensidad de sequía (IIS) es usado para evaluar el desempeño de todas las líneas con respecto a ambas condiciones de humedad y determinar el valor de intensidad de sequía en el experimento evaluado (Fischer and Maurer, 1978).

$\mathrm{IIS}=\frac{1-\mathrm{X}_{\mathrm{d}}}{\mathrm{X}_{\mathrm{p}}}$ 
Donde: $\mathrm{X}_{\mathrm{d}} \mathrm{y} \mathrm{X}_{\mathrm{p}}$ son el promedio de rendimiento de todas las líneas y controles bajo condiciones de estrés hídrico y no estrés, respectivamente.

\section{Resultados}

\section{Caracterización molecular}

La caracterización molecular de las líneas de frijol PS-avp1 confirmó la presencia del gen avp1 de acuerdo al tamaño esperado de un fragmento del gen (630 pb) (Figura 1A). Estas líneas fueron analizadas por sus niveles de expresión transcripcional relativa con valores absolutos variables para cada una de las líneas evaluadas (Figura 1B). No se observó expresión cuantitativa de algún gen endógeno homólogo a la secuencia de avp1, en las plantas no modificadas.
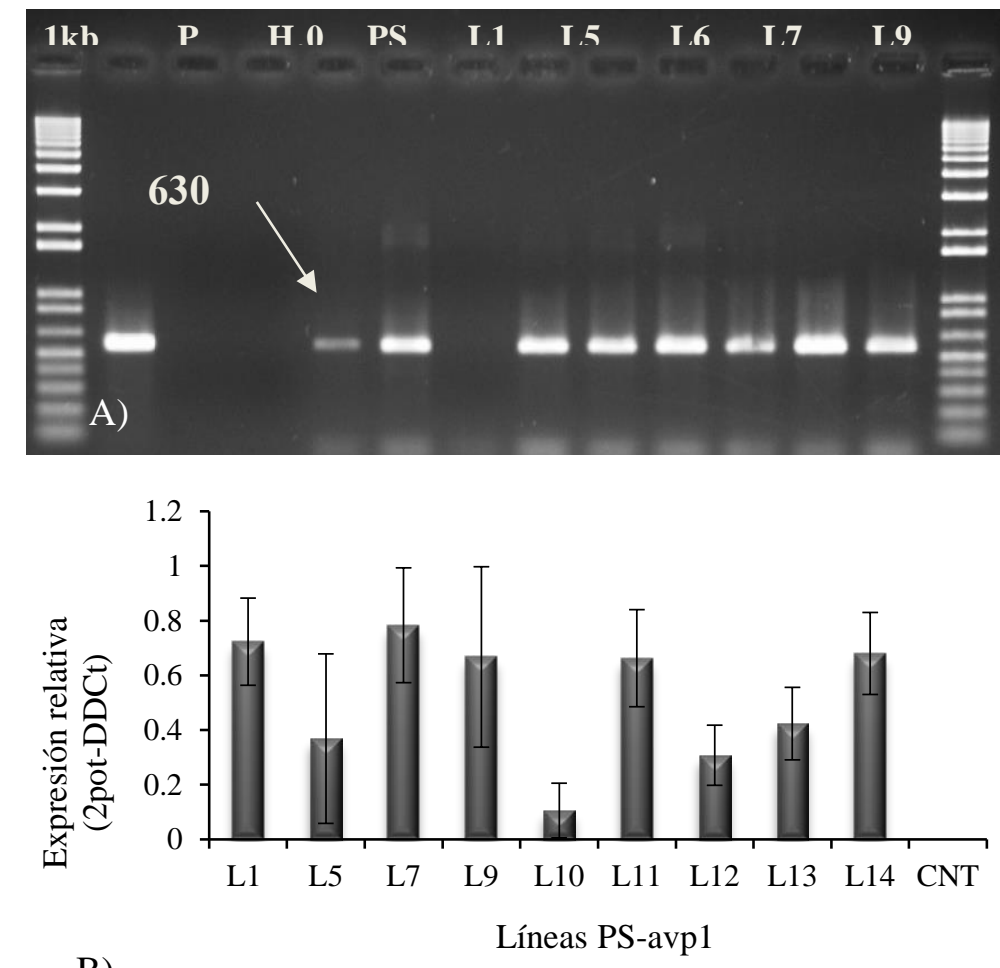

B)

Figura 1. A) Amplificación del gen avp1 por PCR punto final de las líneas homocigotas de frijol cv. Pinto Saltillo. Líneas PS-avp1= L1 a L14; P= plásmido con el gen PS-avp1; H2O, agua sin ADN; PS= frijol cv. Pinto Saltillo no transformado; $M=$ marcador de peso molecular de ADN 1Kb Plus; y B). Expresión transcripcional relativa de avp1 en líneas PS-avp1 y plantas PS. Cada valor corresponde al promedio de diez plantas y tres réplicas de reacción por PCR cuantitativo.

\section{Índice de intensidad de sequía}

El índice de intensidad de sequía (IIS) obtenido bajo condiciones de invernadero fue de 0.78 una de las intensidades de sequía más severas hasta hora reportadas. 


\section{Variables fisiológicas}

La tasa fotosintética en la mayoría de las líneas PS-avp1 fue de 1.4 a 3.1 veces más alto que las plantas PS, bajo estrés hídrico (Figura 2A). El incremento de la tasa fotosintética correlaciona con el rendimiento en la mayoría de las líneas PS-avp1, excepto para L5 que exhibió la tasa fotosintética más baja que L12; sin embargo, ambas tuvieron rendimiento similar bajo estrés (13 g) (Cuadro 1).

Cuadro 1. Relación de rendimiento, biomasa, componentes fenológicos y de partición de plantas de frijol cv. Pinto Saltillo modificado con el gen avp1 bajo estrés hídrico.

\begin{tabular}{|c|c|c|c|c|c|c|c|c|c|c|c|}
\hline Características & L1 & L5 & L7 & L9 & L10 & L11 & L12 & L13 & L14 & $\begin{array}{c}\text { PS } \\
\text { estrés }\end{array}$ & $\begin{array}{l}\text { PS } \\
\text { riego }\end{array}$ \\
\hline \multirow{4}{*}{$\begin{array}{c}\text { Rendimiento } \\
(\mathrm{g}) \\
\text { Hoja }\end{array}$} & 101 & 13.4 & 12.1 & & & & & 8.4 & & 8.4 & \\
\hline & $\pm 0.4 \mathrm{~b}^{*}$ & $\pm 0.2 \mathrm{~d}$ & $\pm 0.4 \mathrm{c}$ & $\pm 0.3 \mathrm{~d}$ & $\pm 0.5 \mathrm{c}$ & $\pm 0.4 \mathrm{e}$ & $\pm 0.4 \mathrm{~d}$ & $\pm 0.2 \mathrm{a}$ & $\pm 0.3 \mathrm{~d}$ & $\pm 0.2 \mathrm{a}$ & $\pm 0.2 \mathrm{~b}$ \\
\hline & 5.1 & 8.4 & 9 & 3.9 & 4.3 & 7.9 & 10.4 & 12.7 & 7.3 & 10.3 & 6.7 \\
\hline & $\pm 0.18 \mathrm{~b}$ & $\pm 0.18 \mathrm{~d}$ & $\pm 0.26 \mathrm{e}$ & $\pm 0.24 \mathrm{a}$ & $\pm 0.22 \mathrm{a}$ & $\pm 0.18 \mathrm{~d}$ & $\pm 0.23 \mathrm{e}$ & $\pm 0.16 \mathrm{f}$ & $\pm 0.14 \mathrm{~d}$ & $\pm 0.4 \mathrm{e}$ & $\pm 0.15 \mathrm{c}$ \\
\hline \multirow{2}{*}{ Tallo } & 9.7 & 12.6 & 19 & 8.4 & 8.2 & 14.4 & 17.1 & 20.5 & 14 & 14.9 & 8.9 \\
\hline & \pm 0.19 & $\pm 0.2 \mathrm{c}$ & $\pm 0.23 \mathrm{e}$ & $\pm 0.28 \mathrm{a}$ & $\pm 0.21 \mathrm{a}$ & $\pm 0.17 \mathrm{~d}$ & $\pm 0.21 \mathrm{e}$ & $\pm 0.18 \mathrm{e}$ & \pm 0.1 & $\pm 0.23 \mathrm{~d}$ & $\pm 0.19 \mathrm{a}$ \\
\hline \multirow[t]{2}{*}{ Vaina } & 2.1 & 2.7 & 2.7 & 3.4 & 1.7 & 3.4 & & 0.8 & 3.4 & 3.8 & 2.4 \\
\hline & $\pm 0.1 \mathrm{~b}$ & $\pm 0.06 \mathrm{c}$ & $\pm 0.08 \mathrm{c}$ & $\pm 0.13 \mathrm{~d}$ & $\pm 0.12 \mathrm{a}$ & $\pm 0.10 \mathrm{~d}$ & $\pm 0.16 \mathrm{e}$ & $\pm 0.07 \mathrm{a}$ & \pm 0.1 & $\pm 0.2 \mathrm{~d}$ & $\pm 0.07 \mathrm{~b}$ \\
\hline \multirow[t]{2}{*}{ Raíz } & 16.8 & 12.7 & 22.7 & 9.82 & 26.9 & 15 & 13.2 & 34.8 & 28.8 & 20.2 & 12.4 \\
\hline & $\pm 1.19 \mathrm{c}$ & $\pm 0.71 \mathrm{~b}$ & $\pm 1.18 \mathrm{~d}$ & $\pm 1.32 \mathrm{a}$ & $\pm 1.5 \mathrm{e}$ & $\pm 0.68 \mathrm{c}$ & $\pm 0.50 \mathrm{~b}$ & $\pm 0.97 \mathrm{f}$ & \pm 0 . & $\pm 0.8 \mathrm{~d}$ & $\pm 0.1 \mathrm{~b}$ \\
\hline Total & 33.89 & 36.53 & 53.64 & 25.67 & 41.31 & 40.82 & 45.44 & 68.89 & 54 & 49.2 & 30.4 \\
\hline \multirow[t]{2}{*}{ TCB } & & & & & & & & & & 0. & 0.35 \\
\hline & $\pm 0.1 \mathrm{a}$ & $\pm 0.1 \mathrm{a}$ & $\pm 0.1 \mathrm{~b}$ & $\pm 0.07 \mathrm{a}$ & & & & & & $\pm 0.1 \mathrm{~b}$ & $\pm 0.1 \mathrm{a}$ \\
\hline \multirow[t]{2}{*}{ TCE } & 0.13 & 0.17 & 0.16 & 0.18 & 0.14 & 0.21 & 0.17 & 0.11 & 0.1 & 0.13 & 0.09 \\
\hline & $\pm 0.09 a$ & $\pm 0.05 \mathrm{a}$ & $\pm 0.02 \mathrm{a}$ & $\pm 0.01 \mathrm{~b}$ & $\pm 0.09 \mathrm{a}$ & $\pm 0.05 \mathrm{~b}$ & $\pm 0.08 \mathrm{a}$ & $\pm 0.05 \mathrm{a}$ & $\pm 0.03 \mathrm{a}$ & $\pm 0.1 \mathrm{a}$ & $\pm 0.01 \mathrm{a}$ \\
\hline \multirow[t]{2}{*}{ TCS } & & & 0.31 & 0.38 & & 0.42 & 0.34 & 0.21 & 0.39 & 0.27 & 0.19 \\
\hline & & $\pm 0.1 \mathrm{a}$ & & $\pm 0.1 \mathrm{a}$ & & & $\pm 0.1 \mathrm{a}$ & & & \pm 0 . & $\pm 0.02 \mathrm{a}$ \\
\hline \multirow[t]{2}{*}{ IC } & & 0.37 & & 0.56 & & & 0.29 & & & & 0.28 \\
\hline & $\pm 0.09 \mathrm{c}$ & $\pm 0.1 \mathrm{c}$ & $\pm 0.03 \mathrm{c}$ & \pm 0.09 & \pm 0.06 & $\pm 0.09 \mathrm{~d}$ & $\pm 0.03 \mathrm{c}$ & $\pm 0.04 \mathrm{a}$ & $\pm 0.03 \mathrm{c}$ & $\pm 0.09 \mathrm{~b}$ & $\pm 0.2 \mathrm{c}$ \\
\hline \multirow[t]{2}{*}{ FRD } & 0.6 & 0.74 & 0.45 & 1.13 & 0.55 & 0.81 & 0.58 & 0.24 & 0.56 & 0.34 & 0.64 \\
\hline & $\pm 0.2 b$ & $\pm 0.2 \mathrm{~b}$ & $\pm 0.2 \mathrm{~b}$ & $\pm 0.2 \mathrm{c}$ & $\pm 0.2 b$ & $\pm 0.2 b$ & $\pm 0.2 \mathrm{~b}$ & $\pm 0.2 \mathrm{a}$ & $\pm 0.2 \mathrm{~b}$ & $\pm 0.07 \mathrm{a}$ & $\pm 0.08 \mathrm{~b}$ \\
\hline \multirow[t]{2}{*}{ G } & 10.24 & 11.82 & 11.22 & 12.27 & 10.86 & 13.09 & 11.7 & 9.32 & 12.53 & 9.35 & 10.39 \\
\hline & $\pm 1.4 \mathrm{ab}$ & $\pm 1.8 \mathrm{~b}$ & $\pm 2.3 \mathrm{ab}$ & $\pm 2.7 \mathrm{ab}$ & $\pm 1.7 \mathrm{~b}$ & $\pm 2.4 \mathrm{~b}$ & $\pm 1.5 b$ & $\pm 0.9 \mathrm{a}$ & $\pm 2.5 \mathrm{~b}$ & $\pm 0.25 \mathrm{a}$ & $\pm 0.1 \mathrm{~b}$ \\
\hline
\end{tabular}

Rendimiento $\left(\mathrm{g} 10\right.$ plantas $\left.^{-1}\right) ; \mathrm{TCB}=$ tasa de crecimiento de biomasa $\left(\mathrm{g} \mathrm{m}^{-2} \mathrm{~d}^{-1}\right)$; TCE $=$ tasa de crecimiento económico $\left(\mathrm{g} \mathrm{m}^{-2} \mathrm{~d}^{-1}\right)$; TCS $=$ tasa de crecimiento de semilla $\left(\mathrm{g} \mathrm{m}^{-2} \mathrm{~d}^{-1}\right) ; \mathrm{IC}=$ índice de cosecha; FRD = fuerza relativa de demanda; $\mathrm{G}=$ rendimiento medio geométrico; ${ }^{*}=$ medias dentro de la misma fila seguidas por letras iguales, no son significativamente diferentes de acuerdo con la prueba de medias de Tukey $\alpha=0.05$.

La tasa de transpiración y la conductancia estomática de las líneas PS-avp1 sufrieron reducciones similares que las plantas PS bajo condiciones de estrés (Figure 2B y 2C). En las tres variables fisiológicas las plantas en riego mostraron valores superiores a todas las plantas bajo estrés.

\section{Componentes fenológicos}

Los valores de las variables DF fueron similares en todas las líneas PS-avp1 y las plantas PS sin modificar (39 d), por lo que no se encontraron diferencias por transgénesis entre las poblaciones evaluadas. Sin embargo, los días requeridos para lograr la madurez fisiológica (DMF) fueron 
menos en las líneas PS-avp1 y las plantas PS (78 días) bajo condiciones de estrés hídrico comparadas con las plantas PS en riesgo ( $85 \mathrm{~d})$. El efecto del estrés hídrico en la cantidad de días requeridos para el llenado de semilla (DLS) indica que las líneas PS-avp1 exhibieron valores similares ( $38 \mathrm{~d}$ ) comparadas con PS bajo estrés (39 d), pero menor que los días requeridos bajos condiciones de riego (45 d) (Cuadro 1).
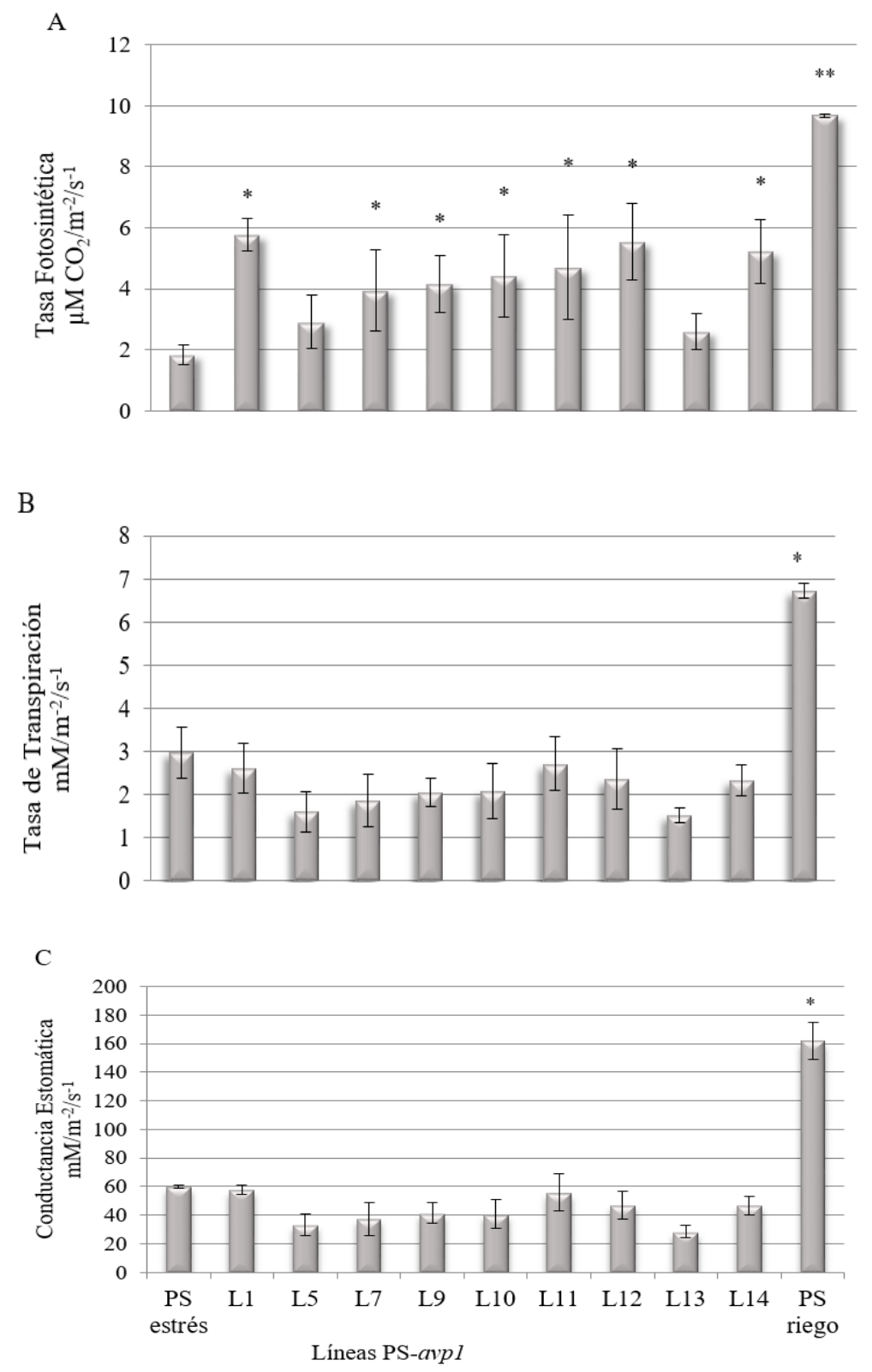

Figura 2. Desempeño de las características fisiológicas de líneas de frijol PS-avp1. A= tasa fotosintética; $B=$ tasa de transpiración; y $C=$ conductancia estomática de líneas transformadas de frijol con el gen avp1 (L1 a L14); PS estrés= frijol Pinto Saltillo no modificado bajo estrés hídrico, PS en riego= frijol Pinto Saltillo no modificado bajo condiciones de riego. Los valores fueron tomados al $20 \%$ del contenido volumétrico de agua en plantas al $50 \%$ de floración. ${ }^{* *}=$ estadísticamente significativo al $1 \% ;{ }^{*}=$ estadísticamente significativo al $5 \%$. 
En este experimento se observó que aun cuando las variables fenológicas en las líneas PS-avp1 bajo estrés hídrico (L5, L7, L9, L10, L11, L12 y L14) tuvieron valores menores comparados con las plantas PS en riego, estas diferencias no afectaron la eficiencia de rendimiento (Cuadro 1).

\section{Rendimiento}

Agronómicamente hablando, la tolerancia a estrés hídrico es percibida como la capacidad de generar rendimiento, no solamente mantener el tejido vegetativo en la planta. Bajo esta premisa, los valores de rendimiento de plantas PS bajo riego (10.4 g 10 plantas $\left.^{-1}\right)$ y estrés hídrico $(8.4 \mathrm{~g} 10$ plantas $^{-1}$ ) fueron empleados para establecer el rango de comparación para el desempeño de las líneas PS-avp1 bajo estrés. Los rendimientos de L1 (10.1 g 10 plantas $^{-1}$ ) y L13 (8.4 g 10 plantas $\left.^{-1}\right)$ fueron los más reducidos de la población de líneas y no fueron estadísticamente diferentes de las plantas PS no modificadas (Cuadro 1).

Es de hacer notar, que siete de las nueve líneas PS-avp1 exhibieron no solamente mejor tolerancia a estrés hídrico que las plantas PS control, sino que sus rendimientos bajo estrés excedieron significativamente los rendimientos de PS bajo condiciones de riego. Los valores de rendimiento logrados por la mayoría de las líneas PS-avp1 mostraron incrementos entre 35\% y $96 \%$ por arriba de las plantas control bajo estrés (Figura 3).

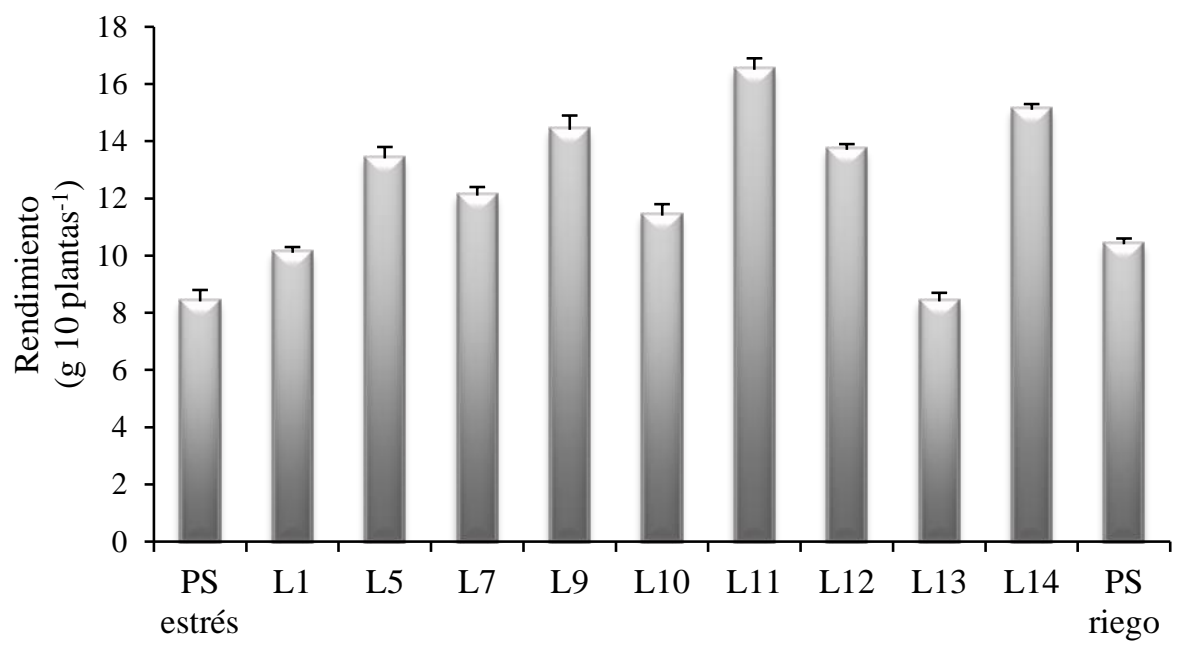

Líneas PS-avp1

Figura 3. Valores de rendimiento de plantas PS-avp1. PS estrés: plantas de frijol cv. Pinto Saltillo no modificadas bajo estrés hídrico, PS riego= plantas de frijol cv. Pinto Saltillo no modificadas bajo riego, L1 a L14= líneas PS-avp1. Cada columna representa el rendimiento de un total de 10 plantas.

\section{Componentes de biomasa}

Los componentes de biomasa mostraron valores variables entre las poblaciones de líneas PS-avp1. Líneas con rendimiento mayor no mostraron mayor biomasa para ninguno de sus componentes; por ejemplo, L5, L9, y L11 tuvieron una biomasa dentro del rango aun debajo de las plantas PS control (Cuadro 1). 
Por otra parte, plantas de la línea L13, tuvieron un rendimiento similar a PS bajo estrés, y mostraron incrementos importantes en biomasa de hoja, tallo y raíz. Líneas L7, L10, L13 y L14 tuvieron mayor biomasa de raíz 21, 55, 118 and 70\%, respectivamente (normalizada con los valores de peso de biomasa de PS bajo estrés hídrico); sin embargo, esto no necesariamente fue reflejado en un mayor rendimiento $(\mathrm{r}=-0.29 ; \alpha \geq 0.05)$. Se observó una correlación negativa entre la biomasa total y el índice de cosecha (IC) $(-0.783 ; \alpha \leq 0.05)$.

Resultados similares fueron obtenidos cuando la biomasa total y la fuerza relativa de demanda (FRD) fueron comparados (-0.81; $\alpha \leq 0.05)$. Las líneas L1 (0.43), L5 (0.46) y L9 (0.33) mostraron una TCB estadísticamente similar a las plantas PS en riesgo (0.35), mientras que L7, L10, L11, L12 y L14 tuvieron valores comparables a las plantas PS bajo estrés hídrico (0.63) ( $\alpha \leq 0.05)$. L13 (0.88) mostró un mayor valor de TCB que el resto de las líneas PS-avp1 y la población PS de plantas control (Cuadro 1).

\section{Índices de partición}

La TCB mostró una correlación significativa con la biomasa de hoja $(r=0.76 ; \alpha \leq 0.05)$, biomasa de tallo ( $\mathrm{r}=0.85 ; \alpha \leq 0.05$ ), y biomasa de raíz ( $\mathrm{r}=0.85 ; \alpha \leq 0.05)$; sin embargo, no mostró correlación con ningún otro de los índices de partición, tales como biomasa de vaina $(\mathrm{r}=-0.25 ; \alpha \leq 0.05) \mathrm{y}$ media geométrica de rendimiento $(\mathrm{G})(\mathrm{r}=-0.307 ; \alpha \leq 0.05)$.

Los valores de la TCE para las líneas L9 y L11 fueron estadísticamente diferentes a las plantas control. El resto de las líneas PS-avp1 no mostraron diferencias estadísticas significativas $(\alpha \leq 0.05)$ (Cuadro 1). En general, los valores absolutos del índice TCS de PS-avp1 también tuvieron valores superiores para la mayoría de las líneas excepto L1 y L13; sin embargo, no se observaron diferencias estadísticas ( $\alpha \leq 0.05)$ (Cuadro 1).

El índice de cosecha (IC) indicó que la línea L13 mostró los valores menores (0.12), aún por debajo de la población de plantas PS bajo estrés (0.21 and 0.28 respectivamente) (Cuadro 1). Las líneas L9 y L11 mostraron los valores mayores ( 0.56 and 0.4 , respectivamente) los cuales coinciden con los valores de rendimiento (Cuadro 1). El resto de las poblaciones de líneas PS-avp1 (L1, L5, L7, L10, L12, y L14), tuvieron valores similares de IC a las plantas PS en riego (0.28) (Cuadro 1).

Los datos de la FRD indicaron que la línea L13 (0.24) tuvo valores cercanos a los valores de las plantas PS bajo estrés (0.34), por otro lado, plantas de la línea L9 (1.13) mostraron diferencias estadísticas $(\alpha \leq 0.05)$, no solo con respecto al resto de las líneas PS-avp1 sino también con respecto a las plantas control PS bajo condiciones de riego y estrés hídrico.

Las líneas PS-avp1 L1, L5, L7, L10, L11, L12 y L14 tuvieron valores de FRD similares $(\alpha \leq 0.05)$ a las plantas PS bajo riego (0.64) (Cuadro 1). Finalmente, el desempeño genotípico (G) de líneas PS-avp1 L5 (11.8), L10 (10.8), L11 (13), L12 (11.7) y L14 (12.5) fue superior al resto de las líneas PS-avp1 y a las plantas PS en riego (10.39). La línea L13 (9.32), mostró un bajo desempeño (G), similar a las plantas PS bajo estrés (9.35); sin embargo, L1 (10.24), L7 (11.22) y L9 (12.27) tuvieron una alta variación de $\mathrm{G}$ dentro de las poblaciones las cuales no las hacen diferentes $(\alpha \leq$ 0.05) a las plantas PS (Cuadro 1). 
El desempeño genotípico $(\mathrm{G})$ de las líneas mostró una alta correlación con el rendimiento $(\mathrm{r}=0.99$; $\alpha \leq 0.05$ ), estableciendo un efecto directo de estrés hídrico sobre la productividad; sin embargo, no se observó correlación entre $\mathrm{G}$ y biomasa total. En contraste, los coeficientes de correlación de los índices de partición TCE, TCS, IC y FRD con G fue alto a significativamente alto ( $r>0.6 ; \alpha \leq 0.05$ ).

\section{Discusión}

El nivel de estrés hídrico es la base para explicar el desempeño fenotípico de las líneas PS-avp1. En este estudio, el índice de intensidad a la sequía (IIS) fue similar al reportado por RamírezVallejo y Kelly (1998), (IIS= 0.63 and 0.78), lo cual define un nivel de estrés extremadamente alto para analizar características agronómicas de tolerancia a este fenómeno.

\section{Características fisiológicas}

Las características fisiológicas analizadas en este estudio mostraron un incremento en la tasa fotosintética en plantas transformadas con el gen avp1 comparadas con las plantas PS control bajo estrés. Este efecto fue previamente reportado por Khadilkar et al. (2016) quien indicó un incremento en los valores de la tasa fotosintética de aproximadamente 1.2 veces mayor en plantas de Arabidopsis thaliana avp1-1.

Esto fue explicado como un mejoramiento en el proceso de fotosíntesis, consistente con las expectativas de un aumento en el transporte de floema debido al incremento de sacarosa y bomba de protones; a través, de simportadores al interior de la membrana plasmática de las células acompañantes del floema para crear una fuerza motriz de protones.

Por otra parte, la reducción en los valores de transpiración y conductancia estomática en poblaciones de líneas PS-avp1 contrasta con lo encontrado por Qin et al (2013), quienes resaltaron que los valores de las tres características fisiológicas mostraron un aumento en plantas modificadas de maní 35Spro: avp1 y sujetas a estrés hídrico (31 d) y salino (60 d). Incrementos similares en fotosíntesis, transpiración y conductancia estomática fueron observados en plantas de algodón 35Spro: avp1 sujetas a estrés salino (Pasapula et al., 2011).

A pesar de estas diferencias, los valores de las variables fisiológicas de las líneas de frijol PS-avp1 bajo estrés hídrico se ubicaron dentro del rango reportado para Pinto Saltillo, bajo condiciones de riego (Chávez-Simental y Álvarez-Reyna, 2012), lo cual indica la eficiencia y plasticidad de las líneas PS-avp1 para su adaptación a condiciones limitadas de agua.

Ramírez-Vallejo and Kelly (1998) reportaron diferencias en las variables fisiológicas a altos valores de IIS (0.78), similar a la información reportada aquí. Plantas de frijol cv. Cacahuate 72 expuestas a tratamientos de estrés y no-estrés mostraron una reducción en su conductancia estomática. Sin embargo, las plantas no mostraron diferencias significativas concernientes a relaciones hídricas cuando fueron comparadas a las plantas bajo riego.

En este estudio el desempeño de la tasa fotosintética es independiente de la conductancia estomática y la tasa de transpiración, esto podría probablemente ser atribuido al ajuste osmótico logrado por el incremento en la expresión de la H+ pirofosfatasa en las líneas PS-avp1 junto con una eficiente translocación de fotosintatos a las semillas. 


\section{Características fenológicas}

Los valores de los componentes fenológicos de las líneas PS-avp1 bajo condiciones de estrés hídrico fueron menores que las reportadas por Acosta-Díaz et al. (2011), es decir, las líneas PSavp1 fueron más eficientes en condiciones de limitación de agua. Esta aceleración a la madurez es generalmente observada cuando hay un periodo prolongado de estrés hídrico durante la etapa reproductiva y no hay condiciones favorables para su recuperación (Samper y Adams, 1985; Schneider et al., 1997; Ramírez-Vallejo y Kelly, 1998; Rosales-Serna et al., 2000).

\section{Conclusiones}

La expresión de la $\mathrm{H}+$ pirofosfatasa recombinante tipo I de Arabidopsis thaliana (avp1) proporcionó protección a estrés hídrico extremo (ISS=0.78) en líneas de frijol PS-avp1. La reducción en la tasa de migración de $\mathrm{CO}_{2}$ y pérdida de vapor de agua a las líneas PS-avp1 durante el estrés hídrico, fue similar a las de las plantas control bajo estrés hídrico; sin embargo, las líneas PS-avp1 mostraron un incremento en la tasa de crecimiento durante el estrés hídrico estableciendo una translocación altamente eficiente de fotoasimilados hacia las semillas, sugiriendo que estas semillas son el órgano destino preferido.

Por otra parte, aun cuando algunas líneas mostraron un incremento en biomasa de raíz, esta característica no proporcionó una ventaja en términos de absorción de agua y mayores rendimientos. Todas las líneas PS-avp1 con valores de rendimiento superiores a las plantas PS en riego, mantuvieron altos valores de índices de partición asociados a la acumulación de materia seca, particularmente IC y FRD. Finalmente, estos resultados muestran las evidencias iniciales de una alternativa prometedora de producción de frijol en regiones agroecológicas con estrés hídrico severo; para lograr esto, se mantiene en perspectiva la liberación al ambiente de las líneas más prometedoras de PS-avp1 para analizar su desempeño bajo condiciones agronómicas.

\section{Agradecimientos}

El autor y autora agradecemos el apoyo a la fuente de financiamiento CONACYT-Guanajuato México, a través del proyecto GTO-2009-C02-118814 'Elementos tecnológicos de transformación genética de frijol'.

\section{Literatura citada}

Acosta, D. E.; Hernández, T. I.; Rodríguez, G. R.; Acosta, G. J. A.; Pedroza, F. J.; Amador, R. M. D. y Padilla, R. J. S. 2011. Efecto de la sequía en la producción de biomasa y grano de frijol. Rev. Mex. Cienc. Agríc. 2(2):249-263.

Acosta, G. J. A. and Rosales, S. R. 1988. Biomasa y sus componentes en variedades indeterminadas de frijol. In: informe de investigación sobre frijol, Durango, México. INIFAP-MSU. 97-196 pp.

Acosta, G. J. A. and Adams, M. W. 1991. Plant traits and yield stability of dry bean (Phaseolus vulgaris L.) cultivars under drought stress. J. Agric. Sci (Cambridge). 117(2):213-219. 
Bao, K. S.; Wang M.; Wu, G. Q.; Xi, J. J.; Zhang, J. L. and Wang C. M. 2008. Overexpression of the Arabidopsis H+-PPase enhanced resistance to salt and drought stress in transgenic alfalfa (Medicago sativa L.). Plant Sci. 176(2):232-240.

Chávez, S. A. and Álvarez, R. V. P. 2012. Ecofisiología de seis variedades de frijol bajo las condiciones climáticas de la Región Lagunera. Rev. Mex. Cienc. Agríc. 3(2):299-309.

CIAT. 2017. Centro Internacional de Agricultura Tropical. http://ciat.cgiar.org/what-wedo/breeding-better-crops/beans/.

Espinosa, H. E.; Quintero, J. A.; Cabrera, B. K. V. and Mora, A. M. A. 2013. Stable and efficient Agrobacterium tumefaciens-mediated transformation of Phaseolus vulgaris. Agrociencia. 47(4):319-333.

Fischer, R. A. and Maurer, R. 1978. Drought resistance in spring wheat cultivars. I: grain yield responses. Aust. J. Agric. Res. 29(5):897-912.

Gamborg, O. L.; Miller, R. A. and Ojima, K. 1968. Nutrient requirements of suspension cultures of soybean root cells. Experim. Cell Res. 50(1):151-158.

Gaxiola, R. A.; Li, J.; Undurraga, S.; Dang, L. M.; Allen, G. J.; Alper, S. L. and Fink, G. R. 2001. Drought- and salt-tolerant plants result from overexpression of the avp1 H+-pump. Proc. Nati. Acad. Sci. USA. 98(20):11444-11449.

Gepts, P. 1998. Origin and evolution of common bean: past events and recent trends. HortSci. 33(7):1124-1130.

Höfgen, R. and Willmitzer L. 1988. Storage of competent cells for Agrobacterium transformation. Nucl. Acids Res. 16(20):9877.

Khadilkar, S.; Yadav, U. P.; Salazar, C.; Shulaev, V.; Paéz, V. J.; Pizzio, G. A.; Gaxiola, R. A. and Ayre, B. G. 2016. Constitutive and companion cell-specific overexpression of avp1, encoding a proton-pumping pyrophosphatase, enhances biomass accumulation, phloem loading, and long-distance transport. Plant Physiol. 170(1):401-414.

Li, B.; Wei, A.; Song, C.; Li, N. and Zhang, J. R. 2008. Heterologous expression of the TsVP gene improves the drought resistance of maize. Plant Biotechnol. J. 6(2):146-159.

Livak, J. and Schmittgen, T. D. 2001. Analysis of relative gene expression data using real-time quantitative PCR and the 2- $\Delta \Delta \mathrm{CT}$ Method. Methods. 25(4):402-408.

Maeshima, M. and Yoshida, S. 1989. Purification and properties of vacuolar membrane protontranslocating inorganic pyrophosphatase from mung bean. J. Biol. Chem. 264(33):20068-20073.

Murray, M. G. and Thompson, W. F. 1980. Rapid isolation of high molecular weight plant DNA. Nucl. Acids Res. 8(19):4321-4326.

Páez, V. J.; Patrón, S. A.; Rodríguez, L. A.; Sánchez, L. J.; Sánchez, G. C.; Valencia, M. P.; Díaz, R. G. and Gaxiola, R. 2011. Plasma membrane localization of the type I H+-PPase avp1 in sieve element-companion cell complexes from Arabidopsis thaliana. Plant Sci. 181(1):2330. doi: 10.1016/j.plantsci.2011.03.008.

Park, S.; Li, J.; Pittman, J. K.; Berkowitz, G. A.; Yang, H.; Undurraga, S.; Morris, J.; Hirschi, K. D. and Gaxiola, R. A. 2005. Up-regulation of a H+-pyrophosphatase (H+-PPase) as a strategy to engineer drought-resistant crop plants. Proc. Nati. Acad. Sci. USA. 102(52):18830-18835.

Pasapula, V.; Shen, G.; Kuppu, S.; Paez, V. J.; Mendoza, M.; Hou, P.; Chen, J.; Qiu, X.; Zhu, L.; Zhang, X.; Auld, D.; Blumwald, E.; Zhang, H.; Gaxiola, R. A. and Payton, P. 2011. Expression of an Arabidopsis vacuolar H+-pyrophosphatase gene (avp1) in cotton improves drought- and salt tolerance and increases fibre yield in the field conditions. Plant Biotechnol. J. 9(1):88-99. 
Qin, H.; Gu, Q.; Kuppu, S.; Sun, L.; Zhu, X.; Mishra, N.; Hu, R.; Shen, G.; Zhang, J.; Zhang, Y.; Zhu, L.; Zhang, X.; Burow, M.; Payton, P. and Zhang, H. 2013. Expression of the Arabidopsis vacuolar H+-pyrophosphatase gene avp1 in peanut to improve drought and salt tolerance. Plant Biotechnol. Rep. 7(3):345-355.

Ramírez, V. P. and Kelly, J. D. 1998. Traits related to drought resistance in common bean. Euphytica. 99(2):127-136.

Rosales, S. R., Ramírez, V. P.; Acosta, G. J. A.; Castillo, G. F. and Kelly, J. D. 2000. Rendimiento de grano y tolerancia a la sequía del frijol común en condiciones de campo. Agrociencia. 34(2):153-165.

Samper, C. and Adams, M. W. 1985. Geometric mean of stress and control yield as a selection criterion for drought tolerance. Ann. Rep. Bean Improv. Coop. 28(1):53-54.

Sánchez, V. I.; Acosta, G. J. A.; Ibarra, P. F. J.; Rosales, S. R. and Singh S. 2004. Registration of Pinto Saltillo Common Bean. Registration by CSSA. Crop Sci. 44(5):1865-1866.

Sarafian, V.; Kim, Y.; Poole, R. J. and Rea, P. A. 1992. Molecular cloning and sequence of cDNA encoding the pyrophosphate-energized vacuolar membrane proton pump of Arabidopsis thaliana. Proc. Nati. Acad. Sci. USA. 89(5):1775-1779.

Schneider, K. A.; Rosales, S. R.; Ibarra, P. F.; Cazares, E. B.; Acosta, G. J. A.; Ramírez, V. P.; Wassimi, N. and Kelly, J. D. 1997. Improving common bean performance under drought stress. Crop Sci. 37(1):43-50.

Terán, H. and Singh, S. P. 2002. Selection for drought resistance in early generations of common bean populations. Can. J. Plant Sci. 82(3):491-497.

Villordo, P. E.; González, C. M. M.; Giraldo, C. P.; Acosta, G. J. A. and Caballero, P. J. 2015. Identification of novel drought-tolerant-associated SNPs in common bean (Phaseolus vulgaris). Front. Plant Sci. 6(546):1-9. 